\title{
Background harmonic distortion measurement at power networks with wind farms
}

\author{
R. Vazquez ${ }^{1}$, M.A. Muñoz ${ }^{1}$, M. Alonso ${ }^{1}$, H. Amaris ${ }^{1}$, C. Alvarez ${ }^{2}$ \\ ${ }^{1}$ Department of Electrical Engineering \\ University Carlos III of Madrid \\ Av. Universidad 30. 28911 Leganés. Madrid (Spain) \\ e-mail: ricardo.vazquez@uc3m.es, monica.alonso@uc3m.es, $\underline{\text { hortensia.amaris@uc3m.es, }}$ \\ ${ }^{2}$ Energy to Quality \\ E2Q \\ Madrid, Spain \\ e-mail: calvarez@energytoquality.com
}

\begin{abstract}
Standard IEC 61400-21 specifies that background voltage distortion must be lower than the threshold limits specified in standards. However, this background distortion information is not used for calculating the real harmonic emission from the wind turbine leading to misleading voltage harmonics assessment from the wind turbine. In this paper a methodology for assessing the real harmonic emission from wind turbines is proposed based on current and voltage measurements where the background harmonic distortion at the Point of Common Coupling is considered.
\end{abstract}

\section{Key words}

Harmonics, Power Quality, Wind Turbines, Grid Integration.

\section{Introduction}

Harmonics have always been a topic of special concern when planning and designing a power system. However, now that the number of renewable energy sources such as wind turbines is only increasing by the day, it is of special interest to study in depth this topic due to the possible failure or damage of sensitive elements that could occur when the distortion of a current or voltage waveform surpasses some limits.

There are measurement and assessment standards such as IEC 61400-21 [1] which provides the procedure to measure and assess the harmonic current emission of wind turbines connected to the grid and IEC standard 61000-430 [2] that specifies the methodology to measure harmonic emissions in power systems. Besides studying voltage and current harmonic emissions from wind turbines, an assessment of harmonic impedance is necessary during harmonic filters design or in those situations where it is necessary to evaluate the power quality of the energy provided by the wind turbine.
In order to quantify the harmonic impedances [3] , a proper evaluation of the voltage and current harmonic components due to the wind power plant at the point of common coupling is needed [3]. The knowledge of the harmonic impedance spectra could be useful for the assessment of the risk of failure caused by overloads or systems resonances. Harmonic impedance spectra could be used to decide whether the connection of a new wind turbine or wind power plant could cause any problem into the power system or not [4].

In this paper, the harmonic impedance analysis from existing wind power plants and its influence on the power systems are studied in order to evaluate if the harmonic emission from a wind power plant are within the limits imposed by the grid operator. In addition, it will be shown both how the consideration of the harmonic background affects the results and the influence of the harmonic angle of the harmonic currents measured in the wind turbine terminals.

The paper is organized as follows. Section II introduces the approach of the study, which describes the main procedure proposed here in order to characterize the background harmonic distortion at the connection point of the wind power plant. In section III, the harmonic background distortion by wind farm is analysed and in section IV, the application of the proposed harmonic voltage process in a wind farm is presented where different scenarios have been considered. Finally, section $\mathrm{V}$ presents the main conclusions of the paper.

\section{Background Harmonic Distortion}

The determination of the background harmonic distortion at the wind farm connection point is vital to study the impact of the wind farm on the grid harmonic distortion 
level. Ref. [4] shows that wind farms offer low-impedance paths to low-frequency harmonics from the grid; thus, lowfrequency harmonics from the grid propagate to the wind farm. The opposite behaviour occurs for the highfrequency components from the wind farm, i.e., they propagate from the wind farm to the grid.

When dealing with wind power installations, network operators perform preliminary analyses of the harmonic emission and propagation in electrical networks using the manufacturer's wind farm model and the available data from electronic power converters. However, in most cases, the data from the wind farm model is not fully detailed or the information from the background harmonic distortion at the PCC is not provided; therefore, it is not possible to accurately determine the impact that the wind farm will have on the harmonic emission in the network.

A widely accepted method to determine the source of harmonic distortion in the grid is based on the direction of power [5], which relies on measurements of the harmonic components at the wind farm connection point (PCC) and determines the harmonic contribution for each of the units or parts that are connected to the same PCC.

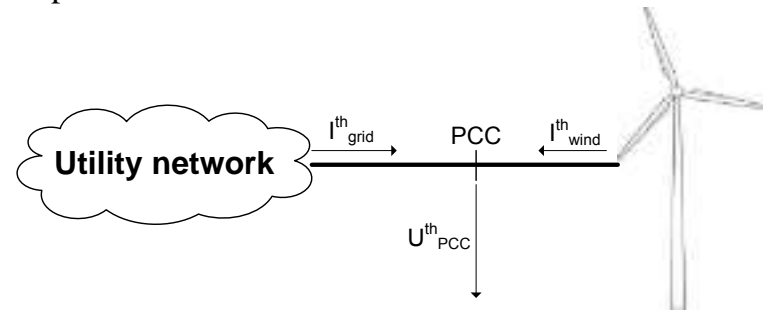

Fig 1. Harmonic propagation from the wind farm and from the grid

According to this method (Fig 1), it would be necessary to measure the voltage harmonics at the PCC $\left(\mathrm{U}_{\mathrm{PCC}}^{\text {th }}\right)$ and the current harmonics injected by the wind turbine $\left(\mathrm{I}_{\text {wind }}^{\mathrm{th}}\right)$ in order to calculate the power harmonics $\mathrm{P}_{\mathrm{PCC}}^{\text {th }}$ that the wind turbine injects into the grid at the connection point as is shown in (1).

$$
\mathrm{P}_{\mathrm{PCC}}^{\mathrm{th}}=\mathrm{U}_{\mathrm{PCC}}^{\mathrm{th}} \cdot \mathrm{I}_{\mathrm{wind}}^{\mathrm{th}} \cos \left(\varphi_{\mathrm{U}}-\varphi_{\mathrm{I}}\right)
$$

In this case, two possible situations can occur:

- If $\mathrm{P}_{\mathrm{PCC}}^{\mathrm{th}}>0$, then the wind turbine is the source responsible of the injection of that "th" harmonic component into the grid.

- On the contrary, if $\mathrm{P}_{\mathrm{PCC}}^{\mathrm{th}}<0$, then the grid is the harmonic source responsible for the injection of that "th" harmonic component to the wind farm.

It has to be taken into account that background harmonics at the connection point present a random behaviour due to variable and random grid operation modes, loads demands and stochastic loads connected to the network. As a result, the measurement process for determining the background harmonic components shall be extended to a long period of time, at least one week, in order to get true and representative results [6].

Fig 2 and Fig 3 show harmonic measurements from the medium voltage side of one wind turbine transformer for two different scenarios:

1) when the turbine was switched off (Fig 2).

2) when the wind turbine was in a continuous operation mode (Fig 3).

Note that some of the measured voltage harmonic components are higher when the turbine is disconnected; thus, it can be deduced that the harmonic currents of the tested turbine at these harmonic frequencies reduce the grid voltage distortion, as is noted in IEC 61400-21 [1].

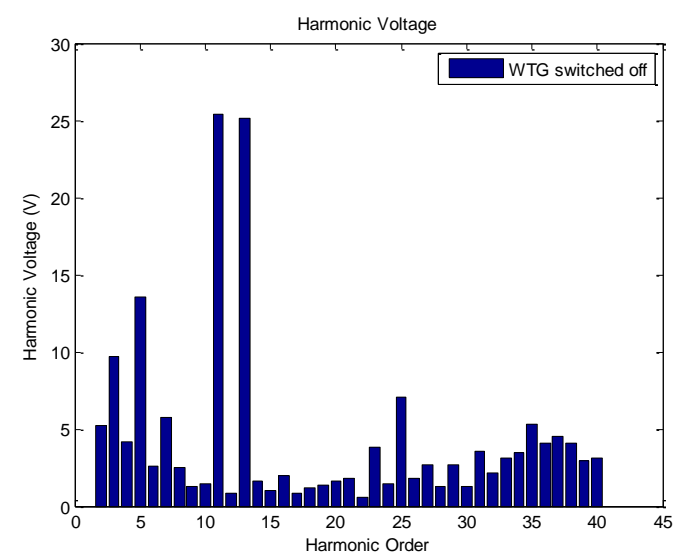

Fig 2. Voltage harmonic subgroup values when the WTG is switched off

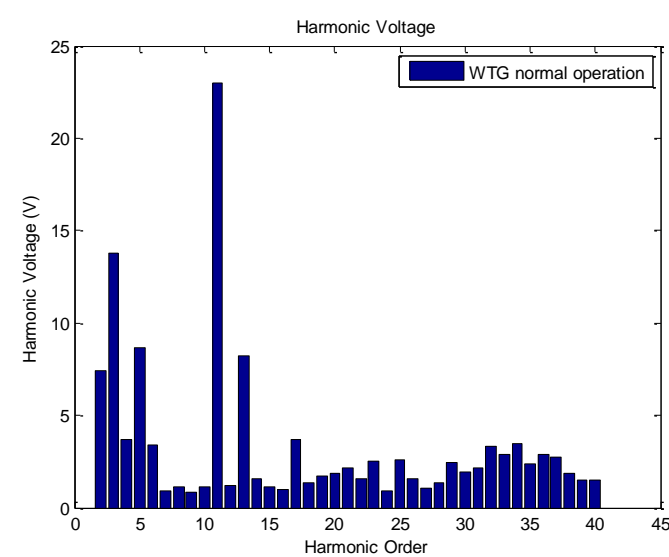

Fig 3. Voltage harmonic subgroup values with the WTG is in operation

Some important information can be drawn from the results:

- For orders $5^{\text {th }}$ and $11^{\text {th }}$ the harmonic voltage values are nearly equal, meaning that the background distortion has a potential influence for these harmonic orders. 
- For the $3^{\text {rd }}$ and $17^{\text {th }}$ order, the harmonic voltages are higher when the wind turbine and its inverter are connected.

- Harmonic orders $7^{\text {th }}$ and $13^{\text {th }}$ show higher harmonic voltage when the wind turbine is disconnected.

Previous conclusions have been applied in several studies related to harmonic assessment. However, the assessments are only valid in those situations where both the grid and the wind farm are injecting different harmonics components, and consequently, a cumulative effect is not possible for each of the individual harmonic components.

\section{Determination of Background Harmonic distortion at wind farms}

The new revision of the international standard IEC 6140021 describes several methods for determining whether the harmonic measurements at the measurement point correspond to harmonics injected by the wind power plant or harmonics produced by the grid (background voltage distortion), and some of the proposed methods are:

- Determine the diurnal variations of the voltage and current harmonics. If the harmonic spectrum shows a clear diurnal variation, then it can be inferred that those harmonic components correspond to harmonic emission from the grid because harmonic emission from the wind turbine depends on power supply variations and does not depend on time of the day.

- The IEC 61400-21 standard recommends measuring a cycle of voltage and current at the Point of Connection with a sampling time of 1 second over at least a oneweek time period in order to detect the background harmonic emission at the point of connection.

- To disconnect the loads or the rest of the wind turbines connected at same point of connection. In this method, measurements and subsequent simulations for wind turbine harmonic assessment shall include a detailed description of the network where the wind power plant is connected. Note that the disconnection of the loads/generators modifies the impedance at the connection point; consequently, this modified impedance value must be updated in the harmonic assessment.

- Voltage harmonics with and without operation of the tested wind turbine. In this situation, measurements of the voltage harmonics are performed before and after the operation of the wind turbine.

In this work, the harmonic distortion in the point of connection due to the wind power plant under study will be analysed considering the point number four of the previous list and through simulations.

\section{Case study}

Harmonic distortion was analysed in a wind farm that was composed of 24 wind generators, with each wind turbine having a power capacity of $2 \mathrm{MW}$, as shown in Fig 4.

The wind farm is composed by three feeders operating at $115 \mathrm{kV}$. There are 2 step capacitors banks rated to 11 Mvar.

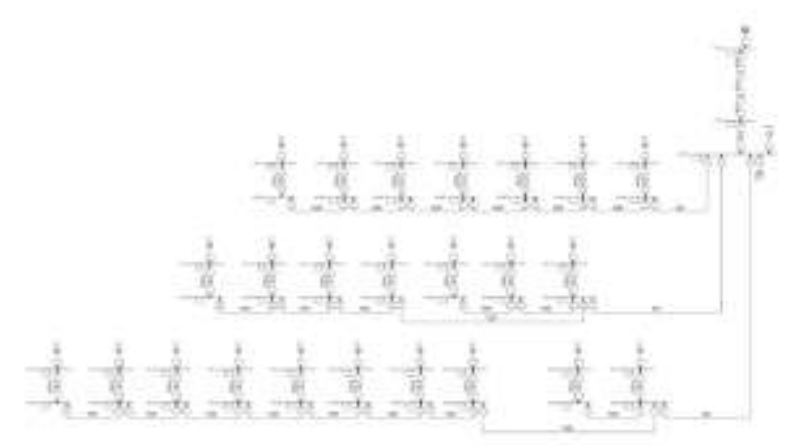

Fig 4. Layout of the wind power plant under study

Current and voltage waveforms were acquired complying with the standard IEC 61400-21[1] that recommends a 10 -cycle window for power systems with a fundamental frequency of $50 \mathrm{~Hz}$. The sampling rate of the measuring equipment was $20 \mathrm{kHz}$ that leads to a resolution of 400 points per cycle. The Discrete Fourier Transform (DFT) is then applied to the measurements using a rectangular window resulting in a good enough frequency resolution $(5 \mathrm{~Hz})$. In order to reduce spectral leakage, harmonic and interharmonic groups and subgroups are obtained as recommended in IEC standard 61000-4-7 [7].

In order to achieve the main objective of this paper, two measurements were carried out:

- Measurements of the harmonic currents of the wind turbine.

- Measurements of the harmonic voltages at the connection point.

Standard IEC 61400-21 [1] states that during the measurement acquisition, data should be sorted in active power bins for different power production levels: $10 \%$, $20 \%, 30 \%, \ldots \ldots, 100 \%$ of the nominal power $\left(P_{n}\right)$ of the wind turbine; consequently 11 active power bins are obtained and the following scenarios have been analysed:

- 12 cases per power factor.

- Reactive Power variation from the wind farm from 0,95 leading to 0,95 lagging. 
In order to quantify harmonic impedance, current and voltage harmonic measurements from the medium voltage side of the wind turbine transformer were used [8], [9]. Then, for each active power bin and harmonic order, harmonic impedance is obtained as is shown in Fig 5:

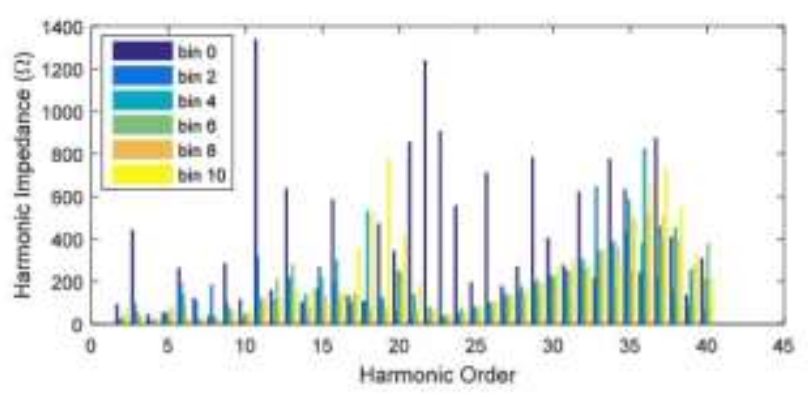

Fig 5. Harmonic impedance for power production levels 0, 20, $40,60,80,100 \%$ of Pn (active power bins $0,2,4,6,8$ and 10)

It can be noted that the impedance obtained from low power production measurements (i.e. bin \#0) is considerably higher at lower harmonic orders (i.e. lower frequencies) compared to other active power bins, reaching its greatest value at harmonic order \#11. On the other hand, impedance calculated from measurements obtained at nominal power (i.e. bin \#10) seems to be lower at the $2^{\text {nd }}-15^{\text {th }}$ harmonic order range to then increase towards its highest value at harmonic order $19^{\text {th }}$; then it decreases to a minimum at the $23^{\text {rd }}$ order to, again, escalate to a local maximum at harmonic order \#37.

In the simulations presented here, the wind power plant has been modelled by implementing as much details as possible. The cables were characterized with direct and inverse impedances and also with zero sequence components, taking into account the variation of the parameters of the cables where crossings were found, etc. The transformers were also detailed in the model and finally the reactive power compensation device was included.

The grid network at the connection point of the wind farm is modelled as a Thevenin equivalent considering the short circuit power declared by the utility.

\section{A. Measurements of the voltage harmonic emission from wind turbines}

Voltage harmonics measured at the connection point, including both background voltage distortion and harmonics from wind farm were also implemented in the model and different situations of the wind farm were considered. In each scenario, reactive power injection from the wind farm was changed in order to consider different wind farm working points such as: $\cos (\varphi)=1$ to a power factor range of 0.95 leading to 0.95 lagging at the connection point.

Considering the harmonic current emission from the wind turbine (Fig 6) and the voltage harmonics measured in the grid, the harmonic voltages at the connection point when the wind farm is working at $\cos (\varphi)=1$ have been calculated and are presented in Fig 7.

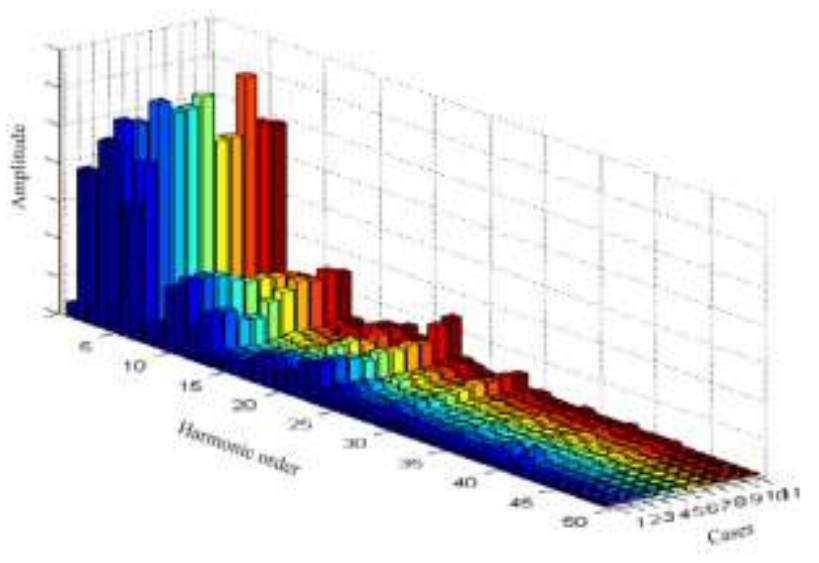

Fig 6. Current harmonics emission from wind turbine $(\cos (\varphi)=1)$

Fig 7 shows the harmonic voltage components for all the studied cases considering not reactive power compensation from the wind farm at the Point of Common Coupling (PCC), $\cos (\varphi)=1$. The cases correspond to the active power injection from the wind farm (case \#1) to full power injection $\left(100 \% \mathrm{P}_{\mathrm{n}}\right)$ which corresponds to case \#12. For each case, the harmonic voltages are shown from $2^{\text {nd }}$ to $50^{\text {th }}$ harmonic order. In the vertical axis, the harmonic amplitude is depicted.

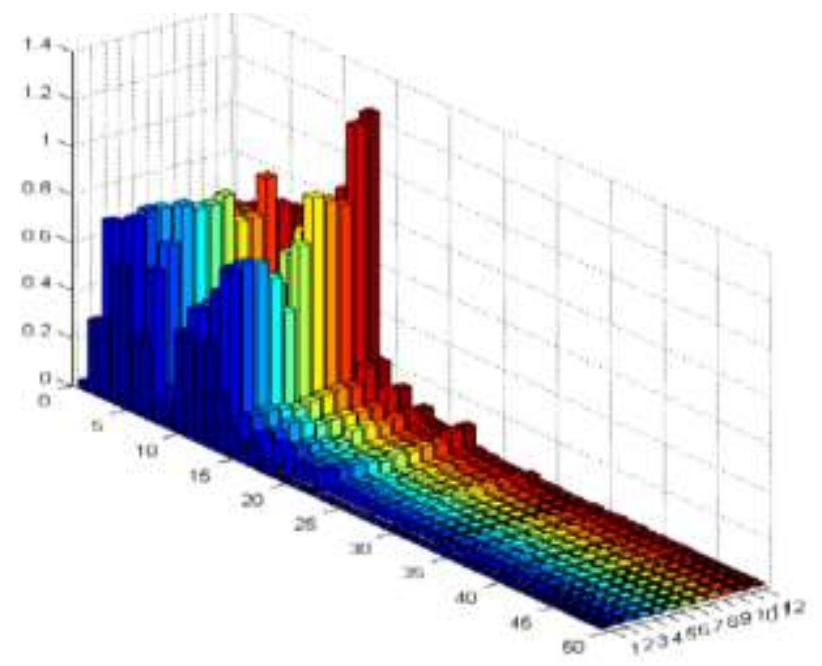

Fig 7. Harmonic voltages at the connection point $\cos (\varphi)=1$

Other cases have been studied where it was considered that the reactive power from the wind farm ranges from a power factor of 0.95 capacitive to inductive. Results are presented in Fig 8 and Fig 9 respectively.

It can be noted that harmonic resonances appear when capacitors are connected (cases: \#1, \#8, \#9, \#10, \#11 \& \#12).

B. Measurements of the voltage harmonic at the PCC without background harmonic distortion 
Harmonic voltage emission from the wind turbine without the voltage background distortion at the PCC can be seen in Fig 10 where the reactive power compensation has not been considered $(\cos (\varphi)=1)$.

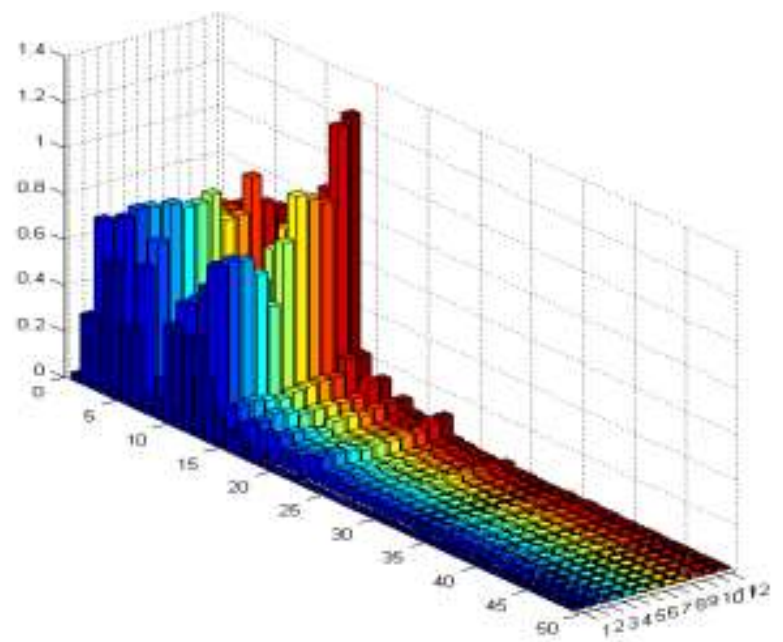

Fig 8. Harmonic voltages evaluated at the connection point with $\cos (\varphi)=0.95$ ind

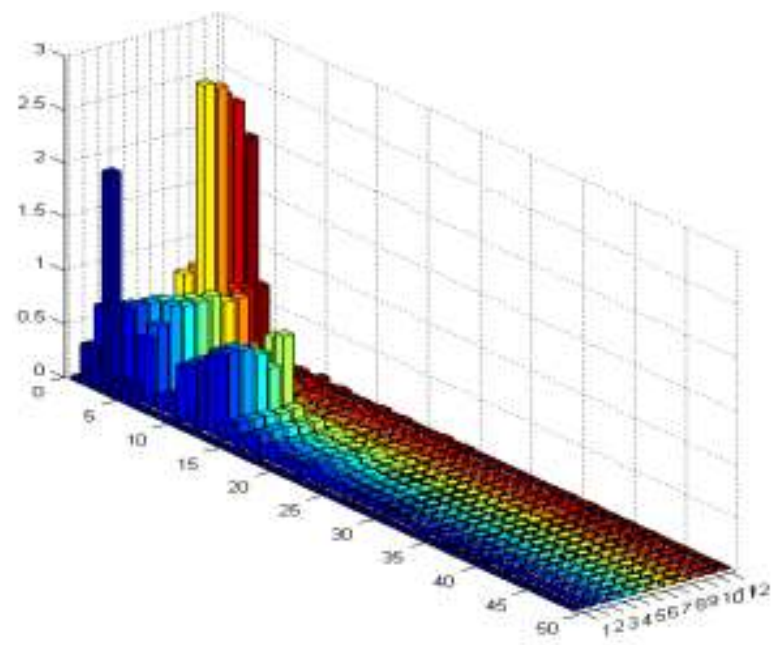

Fig 9. Harmonic voltages evaluated at the connection point with $\cos (\varphi)=0.95$ cap

It can be noted that the biggest differences between the voltage harmonic spectrum from Fig 7 and Fig 10 correspond to case \#1 which corresponds to the case where the wind farm is not injected any active power.

Case \#1 can represent the background harmonic at the PCC. In general, it can be noted that without background harmonic distortion voltage harmonic amplitudes at the PCC (Fig 10) are lower compared to the situation when there is background distortion (Fig 7) for low wind farm generation cases. However, when the wind farm is working to almost full capacity the resonances and harmonic behaviour at the PCC are not affected by the background harmonic components.

If the wind farm is working closely to $\cos (\varphi)=0.95$ cap, due to the reactive power compensation by the capacitor banks at the PCC, the voltage harmonic spectrum at the connection point changes as it is shown in Fig 11.

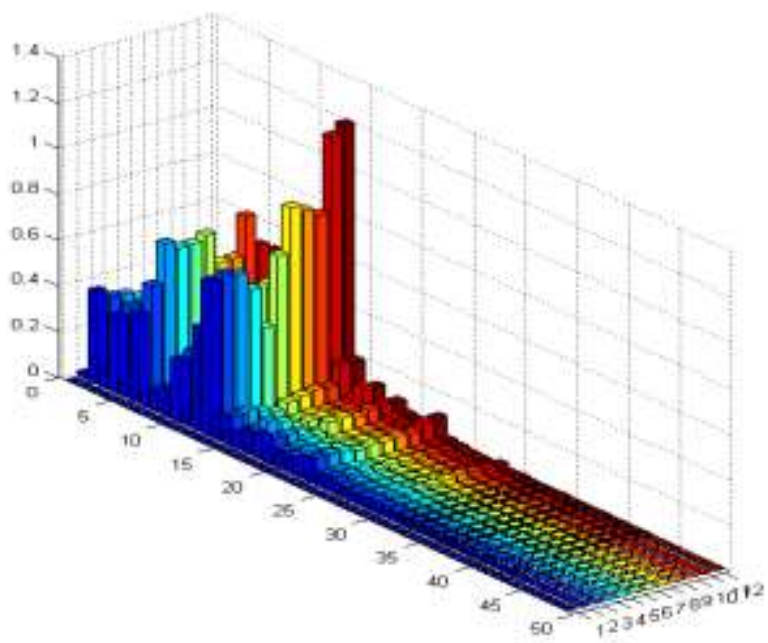

Fig 10. Harmonic voltages emission from the wind farm, $\cos (\varphi)=1$ without background harmonic distortion in the grid

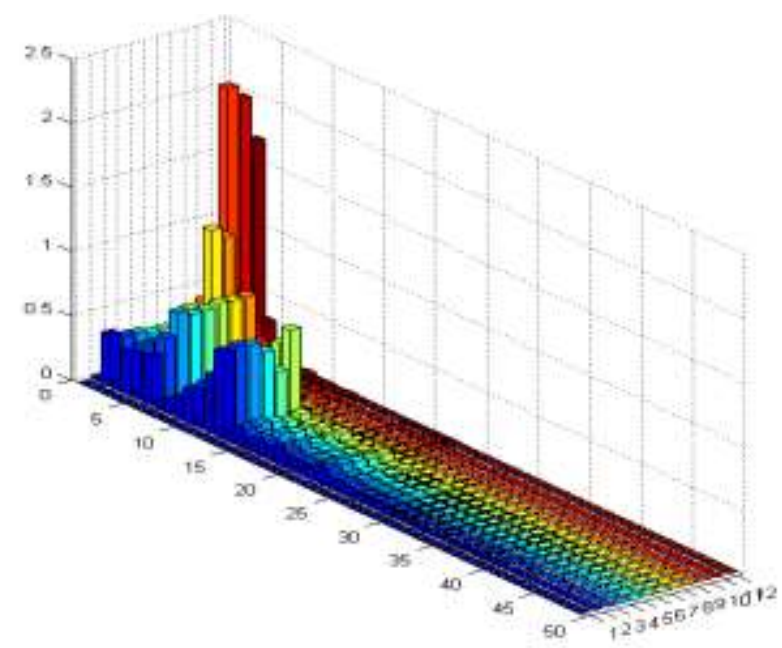

Fig 11. Harmonic voltages emission from the wind farm, $\cos (\varphi)=0.95$ cap without background harmonic distortion in the grid

Comparing Fig 11 (without harmonic background distortion at the PCC) with Fig 9 (with harmonic background distortion at the PCC), it can be noted that resonances corresponding to cases \#10 and \#11 are greatly reduced when there is not voltage distortion at the PCC.

All this cases show that depending on the wind farm emission, some background components from the grid can be increased by the wind farm emission appearing resonances.

\section{Influence of the Harmonic Angle}

In this section, the influence of the angle of the current harmonic is analysed in Fig 12. 
The relevancy of this matter is that in some countries it is mandatory to measure the magnitude of the voltage and current harmonics but the angle measurement is usually not required.

In the following simulations, it can be seen how the angle of the current harmonic has an influence in the global results, just like the angle in the fundamental current has its effect in the results of the normal power flow.

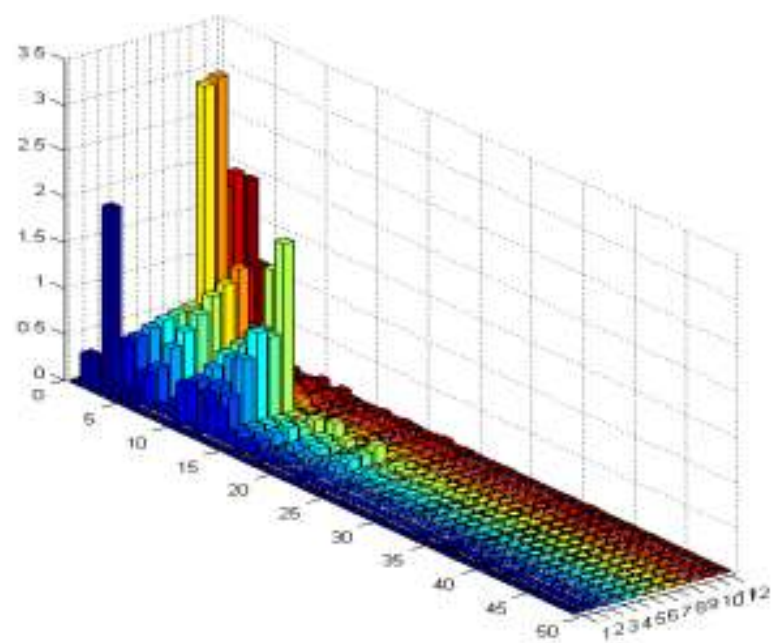

Fig 12. Influence of the harmonic current angle at the Harmonic voltages evaluated at the connection point with $\cos (\varphi)=0.95$ cap

\section{Conclusion}

Standard IEC 61400-21 based in IEC 61000-4-7 addresses the emission of harmonics from wind turbines. In this paper a methodology for assessing the real harmonic emission from wind turbines is proposed based on current and voltage measurements where the background harmonic distortion at the Point of Common Coupling is considered. Simulation studies based on measurements are shown and the influence in certain parameters show that the harmonic voltages assessed at the connection point depends on the harmonic background level at the connection point and on the angle of the harmonic currents of the wind turbines.

In order to assess the voltage harmonics correctly, the measurement at the point of connection must be performed close to the wind power plant installation and the angle of the current harmonics must be included in the power quality assessment.

\section{References}

[1]. IEC 61400-21 ed.2. "Wind turbines - part 21: Measurement and assessment of power quality characteristics of grid connected wind turbines", tech. rep. ed.2, International Electrotechnical Commission, IEC. 2008.
[2]. IEC 61000-4-30, Electromagnetic Compatibility (EMC) Part 4-30: Testing and measurement techniques - Power quality measurement methods, 2008

[3]. E. J. Davis, A. E. Emanuel, and D.J. Pileggi. "Harmonic Pollution Metering: Theoretical Considerations". IEEE TRANSACTIONS ON POWER DELIVERY, VOL. 15, NO. 1, JANUARY 2000.

[4]. M.B. Pereira, E.V. Larsen, S.A. Achilles, "Sensitivity Analysis of Waveform Distortion Assessment for Wind Plants". Brazil WindPower Conference and Exposition, 2014.

[5]. W. Xu, X. Liu, and Y. Liu. "An Investigation on the Validity of Power-Direction Method for Harmonic Source Determination". IEEE TRANSACTIONS ON POWER DELIVERY, VOL. 18, NO. 1, JANUARY 2003, pp 214 219.

[6]. T. Yingjie, Y. Xingang, G. Qinchang, P. Aiqiang, "Background Harmonic Voltage Test and Evaluation". 2010 China International Conference on Electricity Distribution. 2010.

[7]. IEC 61000-4-7, Electromagnetic compatibility (EMC) Part 4-7: Testing and measurement techniques - General guide on harmonics and interharmonics measurements and instrumentation, for power supply systems and equipment connected thereto, 2002.

[8]. Yao Xiao; Maun, J.-C.; Mahmoud, H.B.; Detroz, T.; Stephane Do, "Harmonic impedance measurement using voltage and current increments from disturbing loads," Harmonics and Quality of Power, 2000. Proceedings. Ninth International Conference on , vol.1, no., pp.220,225 vol.1, 2000.

[9]. O. Caubet, S. Ratés. "Site evaluation of harmonics distortions from modern wind turbines based on voltage source and harmonic impedances models" EWEA 2015. 